\title{
Effects of stimulus duration on processing lateralized faces
}

\author{
JOSEPH B. HELLIGE and JON E. JONSSON \\ University of Southern California, Los Angeles, California
}

\begin{abstract}
Observers indicated whether a single probe face presented to the left or right visual field was contained in a positive set of five male faces. In one task the distractor (i.e., negative) stimuli were also male faces, and in another task the distractor stimuli were female faces. For both tasks, reducing stimulus duration from $200 \mathrm{msec}$ to $20 \mathrm{msec}$ increased the percentage of errors, but for neither the percentage of errors nor the reaction time was there any stimulus duration $\times$ visual field interaction. In conjunction with earlier experiments using these same tasks, the results indicate that reducing stimulus duration does not have the same effect on visual laterality as does imposing a visual noise mask. These results pose problems for models predicting that a reduction in stimulus perceptibility per se produces a change in hemispheric asymmetry for face processing.
\end{abstract}

Hellige, Corwin, and Jonsson (1984) examined the lateralized effects of visual noise on tasks that required observers to make a difficult discrimination among a set of similar male faces or that required a male/female discrimination. In the more difficult task, observers indicated on each trial whether or not a single male face was contained in a "positive" set consisting of 5 faces randomly chosen from a set of 10 similar male faces. The single probe face on each trial was presented alone or embedded in visual noise (a grid of small dots). In each of three replications of this task, visual noise increased the error rate to a greater extent when the probe face was presented to the left visual field/right hemisphere (LVF-RH) than when the probe was presented to the right visual field/left hemisphere (RVF-LH). In the less difficult task, observers indicated whether the single probe face was male or female. In this male/female task, visual noise impaired performance to a greater extent on RVF-LH trials than on LVF-RH trials, an effect opposite that for the more difficult male/male task.

These effects of visual noise possibly have something to do with the need to extract relevant visual information from a montage containing both relevant and irrelevant visual information. It is also possible that the results reflect a more general effect of reducing the perceptibility of visual stimuli. According to this second possibility, a manipulation such as reducing stimulus duration should have effects similar to the introduction of visual noise, even though there is no need to filter out irrelevant visual

The research reported here was supported in part by National Science Foundation Grant BNS-8317655 to the first author. Requests for reprints should be sent to J. B. Hellige, Department of Psychology/SGM 704, University Park-MC 1061, University of Southern California, Los Angeles, CA 90089-1061. material. Accordingly, in the present experiment, we examine the effects of stimulus duration on the exact tasks used by Hellige et al. (1984) in their investigation of the effects of visual noise.

\section{METHOD}

\begin{abstract}
The apparatus, stimulus materials, and procedures used in the present experiment are virtually identical to those used by Hellige et al. (1984, Experiment 3) and are not described in detail here. This experiment differed from the Hellige et al. experiment in the following ways.

For each of the two experimental tasks (male/male discrimination vs. male/female discrimination), subjects received 160 experimental trials, separated into two blocks of 80 trials each. Each 80 -trial block contained 10 trials of each of the types defined by the orthogonal combination of stimulus set (positive vs. negative), visual field (LVF vs. RVF) and stimulus duration $(20 \mathrm{msec}$ vs. $200 \mathrm{msec}$ ). Stimulus duration was alternated between $20 \mathrm{msec}$ and $200 \mathrm{msec}$ after each set of 20 trials, with half of the subjects beginning with each duration. Within each set of 20 trials, stimulus set and visual field varied randomly with the restriction that each of the 4 trials defined by the orthogonal combination of the two variables occurred five times.

As in the Hellige et al. (1984) experiment, subjects were told that on each trial a picture of a face would be presented briefly to the LVF or RVF and that their task was to indicate as quickly and as accurately as possible whether the face was a member of a previously memorized positive set of five male faces (requiring a YES response) or not (requiring a NO response). For the male/male task, the stimuli on "negative" trials were male faces, and for the male/female task, the stimuli on "negative" trials were female faces. Subjects responded by releasing one of two telegraph keys (one key labeled YES, the other labeled NO). This response procedure varies from that used by Hellige et al. in that the previous procedure required a key press rather than a key release, but the two response procedures were identical in all other respects.
\end{abstract}

\section{Subjects}

Subjects participating in the present experiment were 10 men and 10 women volunteers from introductory psychology courses at the University of Southern California. All of the observers had normal or correctedto-normal vision in both eyes, were right-handed, and reported fixating on the dot at the appropriate times. 


\section{RESULTS}

For each subject, the percentage of errors and the median reaction time (RT) of correct responses were computed for each of the 16 trial types defined by the orthogonal combination of task (male/male vs. male/female), stimulus duration (20 vs. $200 \mathrm{msec}$ ), stimulus set (positive vs. negative), and visual field (LVF vs. RVF). (The results are collapsed across the two 80-trial blocks because there were no theoretically relevant interactions involving trial blocks.) Preliminary analyses of variance included the sex of the subject and the counterbalancing variables of response-hand arrangement and order of tasks as between-subjects variables. These variables produced no main effects or interactions, so the data were reanalyzed using only the within-subjects variables of perceptual quality, positive/negative, and visual field. For each of the two tasks, similar analyses were performed for both the percentage of errors and RT.

Figure 1 shows the percentage of errors for LVF and RVF trials. The results are shown for stimulus durations of 20 and $200 \mathrm{msec}$ for both the male/male task and the male/female task. As expected from the results of Hellige et al. (1984), the male/male discrimination was much more difficult than the male/female discrimination. For both tasks the percentage of errors was significantly larger with a 20 msec duration than with a $200 \mathrm{msec}$ duration: male/male $F(1,19)=33.24, p<.001$; male/female $F(1$, $19)=7.85, p<.02$. However, for both tasks the effect of stimulus duration was the same for both visual fields,

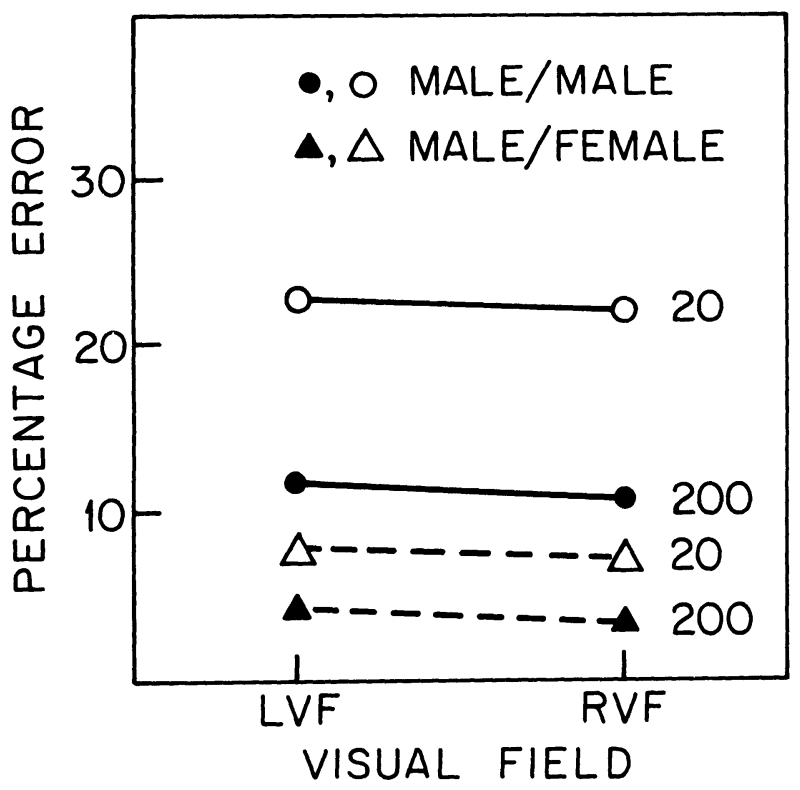

Figure 1. Percentage of errors for left (LVF) and right (RVF) visual field trials for both the male/male and male/female tasks at durations of $200 \mathrm{msec}$ and $20 \mathrm{msec}$. The results are collapsed across the positive-negative variable. so that there was no Stimulus Duration $\times$ Visual Field interaction, $\mathrm{F}<1.0$ for both tasks.

There was one additional statistically significant effect for the male/male task. There were more errors to negative $(26.3 \%)$ than to positive $(19.0 \%)$ stimuli with a $20 \mathrm{msec}$ stimulus duration, with a smaller difference in the opposite direction with a $200 \mathrm{msec}$ stimulus duration (positive mean $=12.5 \%$; negative mean $=9.3 \%), \mathrm{F}(1$, 19) $=6.60, \mathrm{p}<.02$.

The mean of median RTs for the male/male task $(840 \mathrm{msec})$ was considerably higher than the mean of median RTs for the male/female task $(662 \mathrm{msec})$, providing another indication of the difference in difficulty of the two tasks. For the male/male task, the only statistically significant effect for RT was the main effect of positive/negative stimulus type, $\mathrm{F}(1,19)=4.42, \mathrm{p}<.05$, with responses being faster to positive stimuli $(811 \mathrm{msec})$ than to negative stimuli $(868 \mathrm{msec})$.

For the male/female task there was a statistically significant Positive-Negative $\times$ Stimulus Duration interaction, $\mathrm{F}(1,19)=13.13, \mathrm{p}<.002$. The RT to positive (male) faces tended to be shorter to the $20 \mathrm{msec}$ stimulus $(635 \mathrm{msec})$ than to the $200 \mathrm{msec}$ stimulus $(656 \mathrm{msec})$, while the pattern was reversed to the negative (female) faces $(20 \mathrm{msec}$ mean $=685 \mathrm{msec} ; 200 \mathrm{msec}$ mean $=$ $662 \mathrm{msec}$ ). With respect to stimulus duration, the RT results were quite clear. For neither task was there any hint of a main effect of stimulus duration or of a Stimulus Duration $\times$ Visual Field interaction.

\section{DISCUSSION}

The present results, together with those reported by Hellige et al. (1984), indicate that reductions in stimulus duration and the introduction of visual noise do not have the same effects on visual laterality. This is the case despite the fact that both manipulations clearly resulted in impaired performance as measured by an increase in the percentage of errors. Thus, it is difficult to account for the effects of visual noise reported by Hellige et al. (1984) as resulting from simply a reduction in the perceptibility of the face stimuli. Instead, the manner in which the stimuli are perceptually impaired is critical for determining the consequences for visual laterality.

Hellige et al. (1984) suggested that their visual noise masks impaired extraction of local facial details more than the extraction of global characteristics such as the external contour of the face and hairline. One possible reason for the absence of similar stimulus duration $\times$ visual field interactions in the present experiment is that, over the range of durations used, a reduction of stimulus duration impairs the extraction of local and global details to about the same extent. This possibility will be considered in more detail when implications of the present results are considered for a visual spatial frequency model of cerebral hemisphere asymmetry.

Another possibility is that the findings with visual noise masks occur because of hemispheric asymmetry in the ability to distinguish between relevant and irrelevant visual material or to recognize material rendered incomplete by the masks. There is some precedent for this in studies of patients with unilateral brain injury and in patients with callosal section. For example, it has been reported that performance on the embedded figures task is more impaired by left hemisphere than by right hemisphere brain injury (for review see Bradshaw \& Nettleton, 1983; see also Zaidel, 1973). Such results suggest that in some situations the left hemisphere is superior to the right hemisphere in selecting relevant visual features 
from an array that contains irrelevant visual components. On the other hand, similar research with patients has suggested right hemisphere superiority for the recognition of objects from incomplete pictorial representations (e.g., De Renzi, 1978). Thus, the presence of irrelevant visual noise may produce changes in laterality effects for reasons other than a general decrease in stimulus perceptibility.

Regardless of the precise explanation for the different effects of introducing visual noise and reducing stimulus duration, the absence of any stimulus duration $\times$ visual field interactions in the present experiment raises other theoretical questions. In an important series of articles, Sergent $(1982,1983 a, 1983 b)$ has proposed that, at some level of processing beyond the sensory cortex, the left and right hemispheres are biased toward the efficient use of higher and lower ranges of visual spatial frequency, respectively. From this model it follows that the selective elimination of higher spatial frequencies from visual stimuli should interfere more with left hemisphere processing than with right hemisphere processing. Sergent has proposed that an indirect test of the spatial frequency model is provided by manipulation of stimulus duration. As indicated in her reviews, there is reason to believe that visual channels tuned to higher frequencies respond more slowly to stimulation and require higher stimulus energy than do visual channels tuned to lower frequencies. Such characteristics of the visual system suggest that as stimulus processing time is shortened, there is an effective elimination of higher spatial frequencies from processing beyond the sensory cortex. One way to limit stimulus processing time may be to shorten the stimulus duration. If these assumptions are valid, then the spatial frequency model predicts that reductions in stimulus duration should impair processing more on RVF-LH than on LVF-RH trials. In fact, Sergent has reported such stimulus duration $\times$ visual field interactions for both letter comparison and face comparison tasks (Sergent, 1982, 1983a). In view of the theoretical importance of such an interaction, the present results and other failures to obtain this interaction (e.g., Hellige et al., 1984, Experiment 1; Rizzolatti \& Buchtel, 1977, results for females; Safer 1981) merit careful consideration.

One possibility is that the spatial frequency model is fundamentally incorrect, that is, that there is no hemispheric asymmetry in terms of favoring higher versus lower spatial frequencies. Unfortunately, even if this were the case, it would not explain the systematic stimulus duration $\times$ visual field interactions obtained by Sergent and the absence of such effects in the present experiment. Therefore, it is useful to consider other possibilities.

One such possibility is that the reduction of stimulus duration does not always result in the selective degradation of higher frequency channels. There may be some situations in which reducing stimulus duration disrupts the extraction of both high and low spatial frequencies to about the same extent. Such a situation would result in an overall reduction in performance, but an equal reduction for both visual fields. Because local details and global contours would be carried by higher and lower frequency channels, respectively, this would be similar to the possibility discussed earlier that in the present experiment reducing stimulus duration disrupted local and global processing to about the same extent.

In view of the preceding discussion, it is interesting to note that in Sergent's $(1982,1983 a)$ studies reporting a stimulus duration $\times$ visual field interaction, the pre- and postexposure fields have been illuminated to the same level as the stimulus fields. Thus, the briefly presented stimuli are subjected to forward and backward masking by luminance (e.g., Kahneman, 1968). This set of viewing conditions is similar to those used in experiments that have examined the sensitivity of the human visual system to gratings of different spatial frequencies and that have found response time to be faster for lower spatial frequencies (e.g., Breitmeyer, 1975; Breitmeyer \& Ganz, 1977). In contrast, for the present experiment the pre- and postexposure fields were dark, providing no forward or backward masking of the stimuli. Under these conditions, stimulus duration is not a very good index of processing time because of both retinal aftereffects and iconic memory (see Massaro, 1975; Sperling, 1969). To the extent that information can continue to be processed from iconic memory, it may not be important that visual channels tuned to higher frequencies respond more slowly. In effect, it is possible that the selective disruption of higher spatial frequencies is not so great. While this is an admittedly post-hoc and speculative possibility, it is interesting to note that, despite a wide range of other procedural differences, the other face processing experiments that have failed to show any stimulus duration $\times$ visual field interaction also used dark pre- and postexposure fields (Hellige et al., 1984, Experiment 1; Safer, 1981).

In any event, the present results pose problems for any model predicting that a reduction in stimulus perceptibility per se produces a change in hemispheric asymmetry for face processing. While this does not deny that input parameters can contribute to visual laterality in theoretically important ways, it does indicate that the effects of those parameters depend on factors that are not yet completely understood.

\section{REFERENCES}

Bradshaw, J. L., \& Nettleton, N. (1983). Human cerebral asymmetry. New York: Prentice Hall.

BreitMeyer, B. (1975). Simple reaction time as a measure of the temporal response properties of transient and sustained channels. Vision Research, 15, 1411-1412.

Breitmeyer, B., \& GANZ, L. (1977). Temporal studies with flashed gratings: Inferences about human transient and sustained channels. Vision Research, 17, 861-865.

DE RENZI, E. (1978). Hemispheric asymmetry as evidenced by spatial disorders. In M. Kinsbourne (Ed.), Asymmetrical function of the brain. Cambridge: Cambridge University Press.

Hellige, J. B., CoRwin, W. H., \& Jonsson, J. E. (1984). Effects of perceptual quality on the processing of human faces presented to the left and right cerebral hemispheres. Journal of Experimental Psychology: Human Perception and Performance, 10, 90-107.

Kahneman, D. (1968). Method, findings and theory in studies of visual masking. Psychological Bulletin, 70, 404-426.

MASSARO, D. W. (1975). Experimental psychology and information processing. Chicago: Rand McNally.

Rizzolatti, G., \& BUCHTEl, H. A. (1977). Hemispheric superiority in reaction time to faces: A sex difference. Cortex, 13, 300-305.

SAFER, M. A. (1981). Sex and hemisphere differences in access to codes for processing emotional expressions and faces. Journal of Experimental Psychology: General, 110, 86-100.

SERGENT, J. (1982). Methodological and theoretical consequences of variations in exposure duration in visual laterality studies. Perception \& Psychophysics, 31, 451-461.

SERGENT, J. (1983a). The effects of sensory limitations on hemispheric processing. Canadian Journal of Psychology, 37, 345-366.

SERGENT, J. (1983b). Role of the input in visual hemispheric asymmetries. Psychological Bulletin, 93, 481-512.

SPERLING, G. (1969). A model for visual memory tasks. In R. N. Haber (Ed.), Information processing approaches to visual perception. New York: Holt, Rinehart, and Winston.

ZAIDEL, E. (1973). Linguistic competence and related functions in the right cerebral hemisphere of man following comissurotomy and hemispherectomy. Unpublished doctoral dissertation, California Institute of Technology.

(Manuscript received for publication March 19, 1985.) 